\title{
Dispersive shock waves in nonlinear and atomic optics
}

\author{
Anatoly Kamchatnov* \\ Institute of Spectroscopy, Russian Academy of Sciences, 108840, Moscow, Troitsk, Russia
}

\begin{abstract}
A brief review is given of dispersive shock waves observed in nonlinear optics and dynamics of Bose-Einstein condensates. The theory of dispersive shock waves is developed on the basis of Whitham modulation theory for various situations taking place in these two fields. In particular, the full classification is established for types of wave structures evolving from initial discontinuities for propagation of long light pulses in fibers with account of steepening effect and for dynamics of the polarization mode in two-component Bose-Einstein condensates.
\end{abstract}

Dispersive shock waves (DSWs) are universal phenomena of nonlinear physics. They were observed in water waves, plasma, solid state physics, nonlinear optics and dynamics of Bose-Einstein condensates (BECs). As examples of DSWs in the last two fields of research, one can mention observation of DSWs in BEC under action of a repulsive potential [1] and formation of shocks in evolution of light pulses in fibers [2]. In this report, we give a brief review of phenomena that have been recently observed and provide introduction into theoretical methods of their description. The main attention is paid to situations when the standard approach based on the Whitham theory for the NLS (Gross-Pitaevskii) equation is not applicable. For example, long distance propagation of light pulses in fibers can depend essentially on effects of retardation of the medium response on the electromagnetic field. Dynamics of polarization mode in BECs is even more complicated. In both these cases the nonlinear dispersionless velocities are not monotonous functions of the pulse parameters that leads to appearance of new types of wave structures evolving after wave breaking of the pulse. Such wave structures include kinks, trigonometric shocks, combined shocks, and their appearance makes the problem of classification of possible wave patterns much more difficult than in standard and well-known situations described by the NLS equation.

In this talk, we consider approach to the formulated above problem that is based on the developed earlier Whitham theory for the DNLS equation and Landau-Lifshitz equation for magnetics with easy-plane anisotropy. Generally speaking, the suggested method is applicable to nonlinear wave equations that are completely integrable in framework of the Ablowitz-Kaup-Newell-Segur scheme when the inverse scattering transform can be developed. In this case, the reduced finite-gap integration method yields the periodic solutions parameterized by the zeroes of the resolvent $R(v)$ of the polynomial $P(\lambda)$ that defines the solution whereas the zeroes of $P(\lambda)$ play the role of the Riemann invariants of the

\footnotetext{
Corresponding author: kamch@isan.troitsk.ru
} 
corresponding Whitham modulation equations [3]. The specific feature of the new approach is that the mapping of Riemann invariants to resolvent's zeroes is multi-valued and, as a result, one solution of the Whitham equations maps to two different DSW structures [4].

We apply the developed method to consideration of the so-called Riemann problem for evolution of initial discontinuities. The full classification of possible wave structures is established for propagation of long pulses in fibers with account of the steepening effect [5] and for polarization waves in BEC [6,7]. Example of a typical wave pattern is shown in Fig. 1. We present a simple algorithm which permits one to predict parameters of such wave patterns for given initial data at the discontinuity.

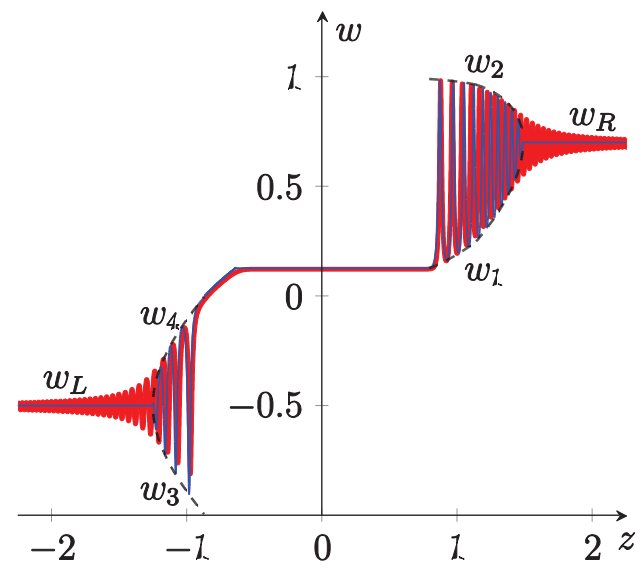

Fig. 1. Example of a wave structure evolving from an initial discontinuity in the polarization mode of two-component BEC dynamics, $w$ is the polarization parameter; $z$ is a coordinate along a trap. Analytical and numerical solutions are shown by blue and red lines, correspondingly.

This work was supported the Program "Topical problems of low temperature physics" of Russian Academy of Sciences.

\section{References}

[1] M.A. Hoefer, M.J. Ablowitz, I. Coddington et al., Phys. Rev. A 74, 023623 (2006)

[2] G. Xu, M. Conforti, A. Kudlinski et al., Phys. Rev. Lett. 118, 254101 (2017).

[3] A.M. Kamchatnov, Nonlinear Periodic Waves and Their Modulations, (World Scientific, Singapore, 2000).

[4] M. Kamchatnov, Y.-H. Kuo, T.-C. Lin, T.-L. Horng, S.-C. Gou, R. Clift, G.A. El, R.H.J. Grimshaw, Phys. Rev. E 86, 036605 (2012).

[5] S.K. Ivanov, A.M. Kamchatnov, Riemann problem for the photon fluid: self-steepening effects (in preparation).

[6] T. Congy, A.M. Kamchatnov, N. Pavloff, Sci. Post. Phys. 1, 006 (2016).

[7] S.K. Ivanov, A.M. Kamchatnov, T. Congy, N. Pavloff, M.A. Hoefer, E. Iacocca, Solution of the Riemann problem for polarization waves in a two-component BoseEinstein condensate (in preparation). 